\title{
Role of MicroRNA-98 in targeting IRS-2, Regulation of Insulin Signaling and Glucose Uptake in the Liver after Bariatric Surgery
}

\author{
Lei Sun", Yong Wang,** \\ ${ }^{1}$ Fuxin Center Hospital, China. \\ ${ }^{2}$ Department of Bariatric Surgery, The Fourth Affiliated Hospital of China Medical University, China.
}

\footnotetext{
How to cite this paper: Lei Sun, Yong Wang. (2020) Role of MicroRNA-98 in targeting IRS-2, Regulation of Insulin Signaling and Glucose Uptake in the Liver after Bariatric Surgery. International Journal of Clinical and Experimental Medicine Research, 4(4), 145-156. DOI: 10.26855/ijcemr.2020.10.005

Received: July 14, 2020

Accepted: August 10, 2020

Published: August 24, 2020

*Corresponding author: Yong Wang, Department of Bariatric Surgery, The Fourth Affiliated Hospital of China Medical University, China.

Email: wy750604@163.com
}

\begin{abstract}
Background: Dysregulation of microRNA expression in various tissues and body fluids has been associated with several diseases, including Type 2 Diabetes Mellitus. In this study, we measured the difference in the expression of microRNA in liver tissue of $\mathrm{db} /+$ and db/db mice. And then, we hypothesized that bariatric surgery could change expression of a certain microRNA which may contribute to the remission of insulin resistance. Methods: $\mathrm{Db} /+$ and $\mathrm{db} / \mathrm{db}$ mice were used to produce bariatric surgery models, then the expression of microRNA in different groups were measured after surgery. With Luciferase assay, we confirmed that microRNA-98 which may be related to the insulin resistance directly targets IRS-2 3'UTR. MicroRNA-98 levels were measured by real-time PCR in $\mathrm{db} / \mathrm{db}$ mice. Insulin signaling was detected with immunoblotting and glucose uptake. Involvement of IRS2 was studied with microRNA mimics and inhibitor. The role of microRNA-98 in vivo was explored with microRNA antagomir. Results: MicroRNA-98 expression in hepatocyte of $\mathrm{db} / \mathrm{db}$ mice was decreased after bariatric surgery as time goes on. We demonstrated that microRNA-98 increased in the liver of $\mathrm{db} / \mathrm{db}$ mice compairing with $\mathrm{db} /+$ mice $(1.85 \pm 0.82$ vs $0.51 \pm 0.27 \mathrm{P}=0.037)$. Overexpression of microRNA-98 decreases IRS2 protein levels $(0.73 \pm 0.05$ relative gene expression unit(a.u) vs $0.52 \pm 0.04$ a.u $\mathrm{P}=0.003 \& 0.26 \pm 0.03$ a.u $\mathrm{P}<0.001$ ) and activity and inhibition of microRNA-98 by antisense oligonucleotides increases IRS2 protein levels $(1 \pm 0.09$ a.u vs $1.28 \pm 0.082$ a.u $\mathrm{P}=0.011 \& 1.52 \pm$ 0.05 a.u $\mathrm{P}=0.001$ ) and activity. Meanwhile, microRNA-98 can affect the uptake of glucose in insulin-resistance cultured hepG2 cells $(21.79 \pm 2.01 \mathrm{mM}$ vs $30.51 \pm 1.19 \mathrm{mM} \mathrm{P}=0.002 \& 28.62 \pm 1.15 \mathrm{mM} \mathrm{P}=0.004 \& 15.09 \pm 1.87 \mathrm{mM} \mathrm{P}=$ 0.009). In addition, inhibition of microRNA-98 by antagomir improves the level of blood glucose in $\mathrm{db} / \mathrm{db}$ mice through mediating expression of IRS2.
\end{abstract}

\section{Keywords}

Microrna, IRS-2, Obesity, Bariatric surgery, insulin resistance

\section{Introduction}

The rapid increase in Type 2 Diabetes Mellitus (T2DM) cases over the past decade has resulted largely from the interaction of genetic susceptibility and environmental factors such as improper diet and sedentary lifestyle [1]. 
T2DM is a metabolic disease characterized by insulin resistance in insulin-sensitive tissues such as white adipose tissue, skeletal muscle fibers and liver tissue, resulting in hyperglycemia [2]. It is preceded by the development of insulin resistance in target tissues, especially the liver which stores glucose after a meal as glycogen or converts excess glucose to fatty acids [3]. Insulin signal transduction is achieved by binding to a specific tyrosine kinase receptor expressed in target cells, which results in increased phosphorylation of a number of substrate proteins, and activation of complex second messenger cascades that regulate glucose and lipid metabolism [4].The recent identification of Insulin Receptor Substrate-2 (IRS-2) as a key factor in the pathophysiology of many of these processes suggests a potentially unifying molecular link underlying the initiation and progression of T2DM [5]. IRS-2 is an important regulatory element for the PI3K-AKT cascade in hepatocytes. IRS-2 is a key molecule in insulin signaling, and is involved in signal transduction between the receptor and phosphoinositide-3-kinase (PI3K) [6, 7]. Systemic disruption of IRS-2 causes insulin resistance in mice that then progress to glucose intolerance and diabetes, due to a combination of pancreatic beta-cell failure, hepatic insulin resistance, and dysregulated hypothalamic signaling [8].

MicroRNAs are a family of endogenous small non-coding RNAs which regulate mRNAs at the post-transcriptional level by partial complementary binding to the 3' untranslated region (3'UTR) of target mRNAs. They either mediate transcript degradation or inhibit translation [9]. The most important determinant of target recognition is the presence of a "seed sequence" capable of pairing to nucleotides 2 through 8 of the microRNA. A single microRNA can potentially bind to a large number of target mRNAs. Multiple microRNAs can also cooperate to fine-tune the expression of the same transcript [10]. Since their discovery, microRNAs have become the focus of intensive research and have been identified as key regulators in governing physiological and pathological processes $[11,12]$. Dysregulation of miRNA expression in various tissues and body fluids has been associated with several diseases, including Type 2 Diabetes Mellitus (T2DM) [13]. In recent study, it showed that microRNA-98 (miR-98) may associated with metabolic sydrome in peripheral blood, possibly regultating glucose and lipid metabolism [14]. MiR-98 was a memerber of Let-7 family, which includes nine slightly different microRNAs. The reaserch demonstrated that Let-7 can regulates glucose metabolism in multiple organs. The member of Let-7 family is considered to have similar functions because they share a common seed region [15]. MicroRNA-98 was found that it decreased in the adventitia and endomembrane in Goto-Kakizaki (GK) rat campared with Wistar rat. It can modulate the expression of tribble 2 (TRB2) which play a important role in diabetic large artery complication [16].

Bariatric sugery is designed to achive and sustain substantial weight loss, effectively prevents and treats type 2 diabetes [17]. Interest has increased rapidly in the mechanism of bariatric surgery that cause weight loss and inprovements in glycaemic control in people with type 2 diabetes. It is still unclear whether bariatric surgery could modulate expression of microRNA to improve insulin resistance. In this study, we sought to test the alter of microRNAs after bariatric surgery and identify a specific microRNA that targets a protein molecule involved in regulation of hepatic insulin sensitivity in vitro and in vivo, and to potentially provide a novel machanism for bariatric surgery to improve insulin resistance

\section{Methods}

\subsection{Animals}

Eight week old male normal (C57BL/Ksj-lepr ${ }^{\mathrm{db}} /^{+}$) and diabetic (C57BL/Ksj lepr ${ }^{\mathrm{db}} /$ lepr $^{\mathrm{db}}$ ) mice [18] were obtained from the Model Animal Research Center of Nanjing University (Nanjing, China). They were kept under standard conditions with $12 \mathrm{~h}$ light/dark cycles and free access to food and water until they were 8 weeks old.

\subsection{Surgery}

Mice were anesthetized with sodium pentobarbital (50mg/kg intraperitoneal injection). The stomach and small intestine were exposed from the abdominal cavity, $15 \mathrm{~cm}$ away from Treitz ligament, and prepared for anastomosed with 6-0 silk sutrer side to side. The pylorus was separated and the 2 parts of the pylorus were dissected and closed. In the sham surgery, the abdominal cavity was opened, but no further surgerical procedures were performded. The abdominal cavity was washed with hated $0.9 \%$ saline and the closed. Penicillin G (50x103 U/kg body mass) was injected subcutneously immediately after surgery to prevent infection. Normal food and regular water were provided after surgery during the recovery period.

\subsection{Target prediction and pathway analysis}

To gain further insight into the role of these miRNAs in the pathogenesis of T2DM, we used the available data- 
bases to search for potential mRNA targets related to insulin resistance. We searched the best three target prediction databases, namely PicTar (http://www.pictar.org/), TargetScan (http://www.targetscan.org/), and microRNA.org (http://www.microrna.org), to look for genes that play key roles in the insulin pathway as potential targets for microRNA-98. We matched these with candidates we found to be repressed by microRNA-98. The remaining putative direct target gene for microRNA-98 was IRS-2, with a single microRNA-98 binding site.

\subsection{Cell culture}

HepG2 cells were obtained from Wuhan Boster Biological Technology, LTD (Wuhan, China). The cells were cultured in DMEM containing $10 \%$ fetal bovine serum at $37^{\circ} \mathrm{C}$, in a $5 \% \mathrm{CO}_{2}$ atmosphere. To induce insulin resistance, the HepG2 cells were pre-incubated in a culture medium containing $1 \times 10^{-6} \mathrm{~mol} / \mathrm{L}$ insulin for $48 \mathrm{~h}$. The uptake of 2-NBDG (2-deoxy-2-[(7-nitro-2,1,3-benzoxadiazol-4-yl) amino]-D-glucose, Invitrogen, CA, USA) was then used as an index to determine insulin resistance.

\subsection{Transfer}

HepG2 cells cultured in DMEM were transfected with miRNA-98 scramble, miRNA-98 mimics (50-100nM) and miRNA-98 inhibitor (50-100nM) (Genepharma, Shanghai, China), using Lipofectamine 2000 (Invitrogen, CA, USA). After 48h, the cells were harvested for analysis by RT-PCR and Western Blot as described below.

\subsection{Real-Time PCR}

The small RNAs were isolated using RNAiso from the Small RNA Kit (TaKaRa) according to the manufacturer's instructions. RT-PCR was done in LightCycler480II using Hairpin-it ${ }^{\text {TM }}$ MicroRNAs Quantitation PCR Kit (Genepharma, Shanghai, China), according to manufacturer's instructions. U6 was used as the endogenous reference RNA. Relative quantification of miRNA expression levels was performed as described above.

\subsection{Western Blotting}

The cells were washed with PBS and lysed with ice-cold RIPA lysis buffer solution (Beyotime, China). Cell lysates were loaded to 6\% and 10\% SDS-PAGE gel and blotted with human anti-IRS-2, Pakt (Ser473), and AKT antibodies (Abcam Ltd, Hong Kong). All antibodies were added at a dilution of 1:1000 in TBST containing 2.5\% non-fat dry milk for $12 \mathrm{~h}$ at $4^{\circ} \mathrm{C}$. The membranes were washed in TBST, followed by induction in goat anti-rabbit secondary IgG antibody with horseradish peroxidase conjugate (Santa Cruz Biotechnology, Inc, CA, USA), using 1:1000 dilutionsin TBST containing 2.5\% non-fat dry milk for $2 \mathrm{~h}$. The membranes were washed again with TBST and visualized with ECL. Relative expression of protein is represented by the quantity of band intensity expressed in relation to that in control samples. Blots were stripped and re-probed using beta-actin (1: 2000) as the internal control. All band images were representatives of at least three independent experiments.

\subsection{Luciferase Assays}

For the luciferase assay, the potential microRNA-98 binding site in the IRS-2 3'UTR was predicted using TargetScan and miRanda. The 3'UTR of the IRS-2 mRNA and a mutant IRS-2 mRNA were synthesized and cloned into the pGLO vector (Promega, United States) downstream from the luciferase stop codon. They were designated as IRS-2 3'UTR-pmirGLO and IRS-2 3'UTR MUT-pmirGLO respectively. Then, 239T cells (70-90\%) were cultured in 24-well plates and co-transfected with eitherIRS-2 3'UTR MUT-pmirGLO or IRS-2 3'UTR-pmirGLO, and either Has-miR-NC(minic control) or Hsa-miR-98 minic using Lipofectamine 2000 (Invitrogen, USA). Forty-eight hours later, the cells were harvested and luciferase activity was measured using a Dual-Luciferase Reporter Assay kit (Promega, USA).

\subsection{Uptake of glucose}

The effect of miRNA-98 and its inhibitor on insulin dependent glucose uptake was assessed by 2-NBDG uptake. HepG2 cells treated with insulin were transfected with miRNA-98 mimic or its inhibitor as described above. After $48 \mathrm{~h}$, the cells were pre-incubated in $0.05 \%$ glucose containing Krebs Ringer Bicarbonate buffer solution (PH7.4 with $2 \% \mathrm{BSA}$ ). After this pre-incubation at $37^{\circ} \mathrm{C}$ for $30 \mathrm{~min}$, the cells were then incubated in the presence or absence of insulin $(100 \mathrm{nM})$ for $10 \mathrm{~min}$. They were then further treated with insulin and 2-NBDG for $2 \mathrm{~h}$ at $37^{\circ} \mathrm{C}$ in glucose-free Krebs Ringer Bicarbonate buffer solution containing 2\% BSA. On termination of incubation, the cells were washed with cold phosphate-buffer saline (PBS), and 2-NBDG uptake into the cells was assayed by FACS analysis. 


\subsection{The function of miRNA-98 in vivo}

To validate whether miRNA-98 inhibition could alleviate diabetic status in vivo, experiments were done in the normal and diabetic $\mathrm{db} / \mathrm{db}$ mice. The animals were divided into three groups: normal $\mathrm{db} /+$ mice injected with scramble; diabetic $\mathrm{db} / \mathrm{db}$ mice injected with scramble; and $\mathrm{db} / \mathrm{db}$ mice injected with antagomir-98(80mg/kg). We subsequently measured the changes in blood glucose levels in the animals. To assess oral glucose tolerance (OGTT), the animals which were injected on the third day were fasted overnight and their serum glucose response to oral administration of $20 \%$ glucose $(2 \mathrm{~g} / \mathrm{kg})$ was measured. Blood was drawn from the tail at $0,30,60,90$ and 120 minutes after administration of glucose. Random blood glucose levels of fed animals were measured at the start of the experiment (day 0), and on each day of the three consecutive days of the experiment. Finally, the animals were euthanized on the next day and liver tissue dissected for analysis. The levels of IRS-2, pAkt and Akt were determined by Western Blot.

\subsection{Statistical analysis}

All experiments were performed three times and data expressed as the mean \pm SEM. The values represent the mean of at least three independent experiments. The statistical significance of the differences was evaluated using the Student's t-test, with $\mathrm{p}<0.05$ considered statistically significant.

\section{Results}

\subsection{Bariatric surgery reduced body weight and improved blood glucose}

The effects of bariatric surgery on weight loss and glycemic control were examined. We detected the weight loss and blood gulucose at 5, 10, 20, 30 days after bariatric surgery in mice. As shown in Table 1, rapid weight loss and decrease in body fat mass by 5 days after surgery about $13.5 \%$, and the body weight continued to decrease at days 10,20, and 30 days after surgery. At the same time, after surgery the blood glucouse decreased gradually and was close to the normal levels. The results demonstrate that bariatric surgery can decrease the weight and body fat mass and improve the blood glucos metablism.

Table 1. Basic parameters before and after bariatric surgery

\begin{tabular}{|c|c|c|c|c|c|c|c|}
\hline & \multicolumn{2}{|c|}{$\mathrm{db} /+$} & \multicolumn{5}{|c|}{$\mathrm{db} / \mathrm{db}$} \\
\hline & Sham & surgery & Sham & post-surgey & post-surgey & post-surgey & post-surgey \\
\hline & & & & 5days & 10 days & 20 days & 30 days \\
\hline \multicolumn{8}{|c|}{ Body mass(g) } \\
\hline Before & $23.4 \pm 0.4$ & $25.3 \pm 0.6$ & $48.2 \pm 1.8^{*}$ & $51.6 \pm 2.5^{*}$ & $50.4 \pm 1.9^{*}$ & $49.4 \pm 1.5^{*}$ & $50.3 \pm 2.3^{*}$ \\
\hline After & $24.6 \pm 0.7$ & $23.2 \pm 1.6^{*}$ & $48.9 \pm 1.2^{*}$ & $44.5 \pm 1.7^{* \# \&}$ & $43.2 \pm 2.7^{* \# \&}$ & $40.8 \pm 1.4^{* \# \&}$ & $41.4 \pm 2.2^{* \# \&}$ \\
\hline \multicolumn{8}{|c|}{ Glucose level(mf/dl) } \\
\hline Before & $94 \pm 8$ & $100 \pm 12$ & $460 \pm 23^{*}$ & $478 \pm 34^{*}$ & $502 \pm 45^{*}$ & $490 \pm 27^{*}$ & $512 \pm 65^{*}$ \\
\hline After & $89 \pm 12$ & $78 \pm 9$ & $240 \pm 14^{*}$ & $189 \pm 28^{* \# \&}$ & $204 \pm 39^{* \# \&}$ & $198 \pm 19^{* \# \&}$ & $178 \pm 24^{* \# \&}$ \\
\hline \multicolumn{8}{|c|}{ Food intake(g) } \\
\hline Before & $4.4 \pm 0.34$ & $4 \pm 0.53$ & $6.6 \pm 0.65^{*}$ & $6 \pm 0.34^{*}$ & $5.8 \pm 0.7^{*}$ & $6.2 \pm 0.64^{*}$ & $5.9 \pm 0.42^{*}$ \\
\hline After & $3.5 \pm 0.27$ & $3 \pm 0.6^{\&}$ & $5.9 \pm 0.47^{*}$ & $4.3 \pm 0.63^{* \# \&}$ & $4 \pm 0.53^{* \# \&}$ & $4.2 \pm 0.36^{* \# \&}$ & $3.9 \pm 0.37^{* \# \&}$ \\
\hline
\end{tabular}

Notes: Bariatric surgery decreased the body mass of mice and improved the glucose level of blood. ${ }^{*} \mathrm{P}<0.05$ compaired with the $\mathrm{db} /+$ sham mice; ${ }^{\#} \mathrm{P}<0.05$ compaired with the $\mathrm{db} / \mathrm{db}$ sham mice; ${ }^{\circledR} \mathrm{P}<0.05$ compared with $\mathrm{db} /+$ and $\mathrm{db} / \mathrm{db}$ mice before surgery.

\subsection{The effect of bariatric surgery on expression of microRNAs in adipoctye of mice}

After 30 days of bariatric surgery, the mice were killed and liver tissues were collected. We examined the expression of some microRNAs which including miR-98. As a result, we found expression of miR-98 was decreased camparing with the sham surgery in Fig1A. Bariatric surgery can significantly improved body mass and insulin resistance, although the mechanism of improved insulin sensitivity remains unclear. May variation of miR-98 in mice contribute to the bariatric surgery to medicating insulin resistance in diabetes? Then next, we wanted to verify whether miR-98 can improved insulin resistance in liver cells. 


\subsection{MiR-98 levels are up-regulated in the hepatocytes of $\mathrm{db} / \mathrm{db}$ mice}

To evaluate miR-98 expression pattern in the hepatocytes of $\mathrm{db} / \mathrm{db}$ mice which are obese and diabetic due to mutation of the leptin receptor gene, we performed RT-PCR. Liver tissue was taken from the animals of each group, namely the normal $(\mathrm{db} /+)$ and the diabetic $(\mathrm{db} / \mathrm{db})$ group. Subsequent analysis revealed a significant 3.6-fold up-regulation of miR-98 expression in the liver of $\mathrm{db} / \mathrm{db}$ mice compared with the control mice (Figure 1B).

We then assessed the status of IRS-2 in hepatocytes from both the normal and the diabetic groups of mice. As shown in Figure 1C \& 1D, the levels of IRS-2 were significantly down-regulated at the transcript and protein level in hepatocytes of the $\mathrm{db} / \mathrm{db}$ mice compared to those of the normal mice, a pattern opposite to the expression of miR-98. This implies that the elevated levels of miR-98 might be responsible for the decreased levels of IRS-2 in the hepatocytes of $\mathrm{db} / \mathrm{db}$ mice.

A

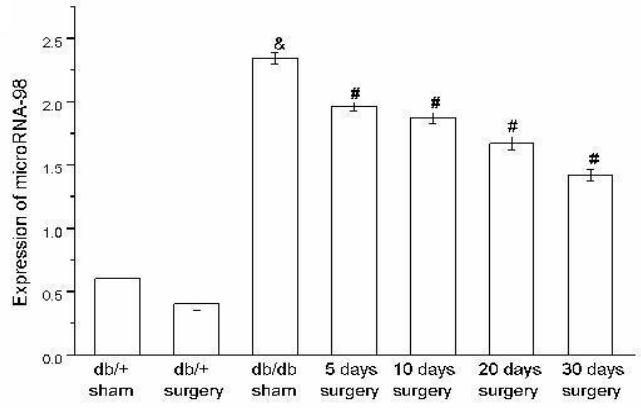

B

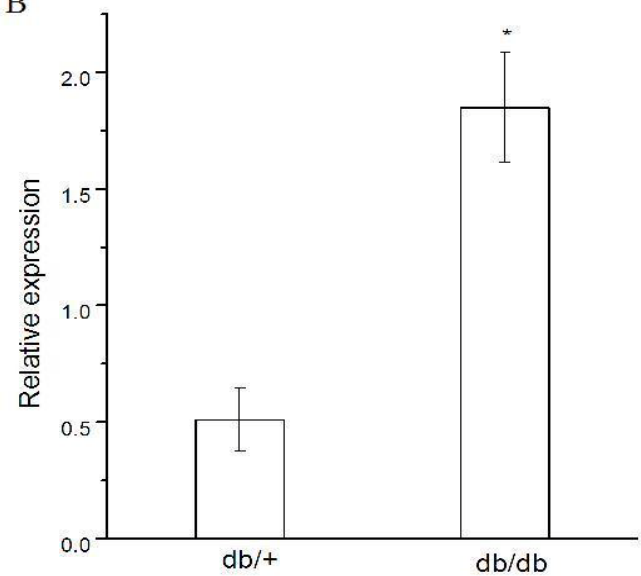

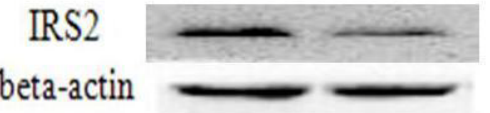

$\mathrm{D}$

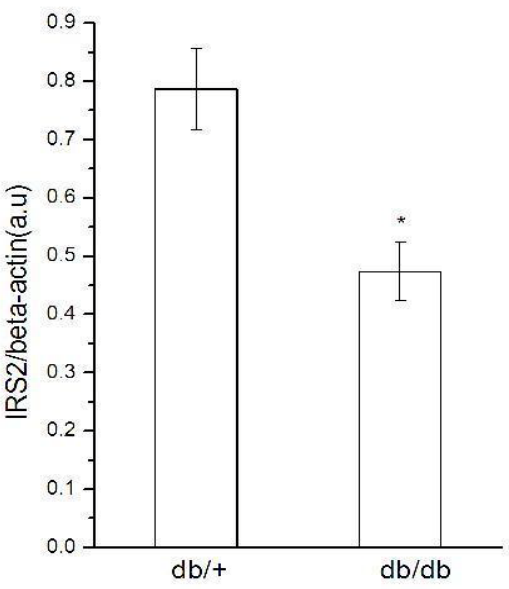

Figure 1. Bariatric sugery reduced the level of microRNA-98 expression in liver cells of dbldb mice. (A) Ildentification of microR-98 as a signature microRNA and IRS2 protein levels were assessed in liver tiusse of db db. (B) Real-Time quantitative PCR analysis of miR-98 levels in heptocyte of $\mathrm{db} /+$ and $\mathrm{db} / \mathrm{db}$. (C) Liver tiuess were lysed and 50 ug protein from each incubation was resolved on SDs-PAGE and evaluated for the levels of IRS2 using anti-IRS2 antibody beta-actin was taken as the loding control. Representative blot for each animal is given and (D) the densitometric analysis is given as following. Each bar represents the mean \pm SEM (n=6 per group). ${ }^{\&} \mathrm{P}<0.05$ compared with $\mathrm{db} /+$ sham surgery; ${ }^{\#} \mathrm{P}<0.05$ compared with $\mathrm{db} / \mathrm{db}$ sham surgery. $* \mathbf{P}<0.05$ vs $\mathrm{db} /+$.

\subsection{Overexpression of miR-98 decreases glucose uptake and impairs insulin sensitivity in HepG2}

To examine whether overexpression of miR-98 could affect insulin sensitivity, a 2-NBDG uptake assay was performed in hepG2. In Figure 2A, insulin treatment alone of hepG2 significantly increase glucose uptake by $17.2 \%$, and overexpression of miR-98 increase insulin-stimulated glucose uptake by $98.7 \%$ compared with the basal levels. MiR-98 overexpression did not increase glucose uptake from the basal level, but significantly decreased insulin-stimulated glucose uptake by $72.6 \%$ compared with the untransduced (Untr) or control groups.

Based on the impact of miR-98 gain of function on glucose uptake, we next test whether miR-98 affected insulin signaling in hepG2. Immunoblotting was performed with hepG2 trasfected with the miR-98 minic or scramble. 
As shown in Figure 2B, overexpression of miR-98 markedly downregulate IRS2 protein levels, whereas overexpression of mutant miR-98 had no significant effect. More importantly overexpression of miR-98 markedly downregulated IRS2 activity and decreased the pAKT (Ser473) which known the downstream protein of IRS2. These data demonstrated that miR-98 overexpression induced hepG2 insulin resistance.

\subsection{MiR-98 targets IRS-2 3’UTR via a miR-98 binding site}

To determine whether miR-98 targets IRS-2 3’UTR, microRNA target-prediction programs miRBase, PicTar and TargetScan were used to identify potential binding sites. Most importantly, among the pathways overrepresented by the targets of miR-98, the type 2 diabetes pathway (corrected $p$ value $=12.3$ ) was the most highly significant pathway. MiR-98 was therefore prioritized for evaluation of a possible role in the pathogenesis of T2DM. We sought to ascertain whether IRS-2 is a direct target for miR-98. The region from 453 to 460 nucleotides on the 3'UTR of IRS-2 gene harbors the binding site for miR-98. Therefore, the pathophysiology of T2DM could potentially be correlated with microRNA-98 expression, which in turn may affect IRS-2 protein levels (Figure 3A).

A

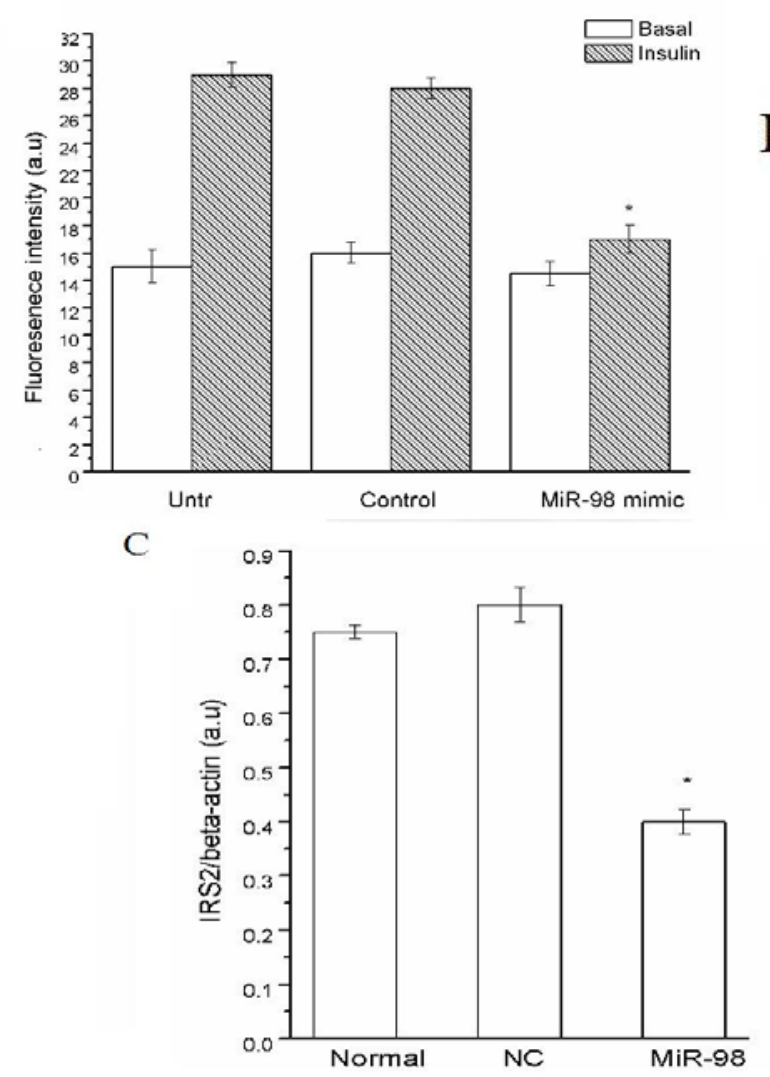

B

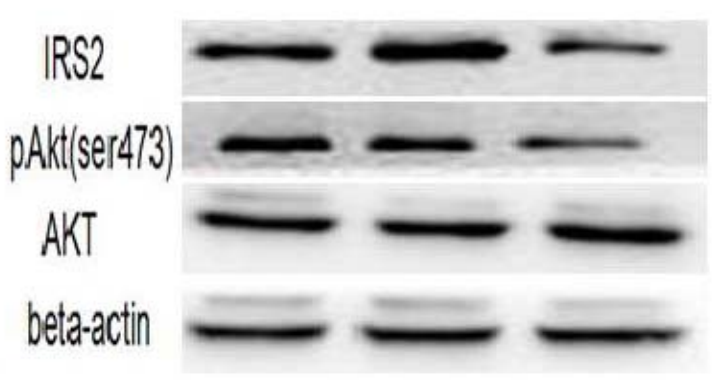

D

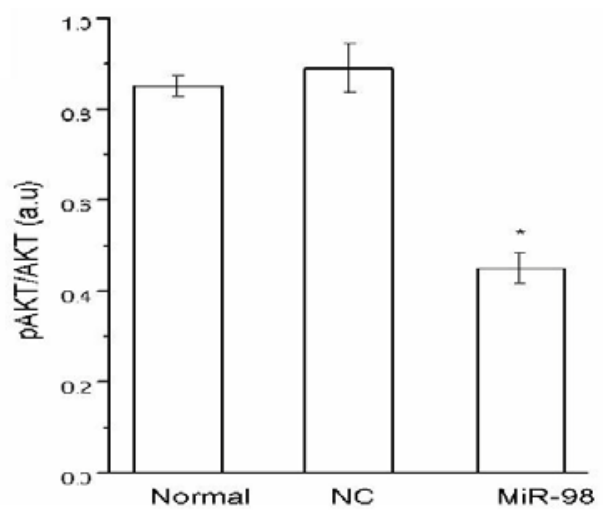

Figure 2. Overexpression of microRNA-98 decreased glucose uptake and impaired insulin sensitivity in hepG2 cells. To indentified miR-98 can effect insulin signaling in the absence of insulin in hepG2 cells, and then cells were transfected with either the mi-NTR (negative control) or miR-98 mimic and without any thing as normal. (A) Effect of microRNA-98 overexpression on 2-NBDG in hepG2 cells in the absence or presence of insulin. (B) After transfection,cells were lysed and lysates (5Oug) were subjected to Western Blot analysis using IRS2, pAkt and Akt antibodies.Beta-actin was taken as the loading control. (C D) The immunoblot intensities for IRS2/beta-actin and pAkt/Akt was quantified by densitometry and expressed in relative ratio where the intensity of negative control was set to one. Values are expressed as means \pm SEM from five independent experiments, ${ }^{*} \mathbf{P}<0.05$.

To investigate whether IRS-2 is responsive to miR-98, the 3'UTRs of human IRS-2 were cloned into pmirGLO vector, downstream to the Renilla luciferase coding sequence, and co-transfected with miRNA mimics into human embryonic kidney 239T (HEK293T) cells (Figure 3B). Indeed, Renilla luciferase activity decreased by $40.2 \%$ compaired with non-targeting control trasfected cells. In addition, site-directed mutagenesis of the seed region abolished the inhibitor effect of miR-98 on Renilla luciferase activity, but miR-98 minic still decreased the repoter gene by 36\% compaired with mutant (Figure 3C \& 3D). These results indicate that miR-98 suppresses gene ex- 
pression by binding to the seed sequence at the 3'UTR of IRS-2. Therefore, IRS-2 may be a direct target for miR-98.

\subsection{MiR-98 over-expression attenuates insulin-induced phosphorylation and activation of AKT, and glucose uptake}

To test whether miR-98 regulates insulin sensitivity, we first measured the effect of miR-98 on insulin signaling in HepG2 cells treated with insulin. We transfected HepG2 cells with micoRNA mimics or negative control, and investigated whether induction of miR-98 directly represses IRS-2 expression. As shown in Figure 4A, Figure 4B, and Figure 4C, the over-expression of miR-98 in HepG2 cells showed a significant dose-dependent reduction in IRS-2 protein levels, and insulin-induced phosphorylation of AKT at Ser473. The induction of insulin resistance by microRNA-98 supports our proposal that inhibition of miR-98 might potentially improve insulin sensitivity. We thus inhibited endogenous expression of miR-98 in HepG2 cells using antisense oligonucleotides to test its resultant effect on insulin sensitivity. In the presence of insulin and inhibition of miR-98, we observed significant up-regulation of IRS-2 levels and insulin-induced phosphorylation of AKT (Figures 4D, 4E \& 4F). These results clearly indicate that induction of miR-98 causes insulin resistance in hepatocytes by reducing the expression of IRS-2.

A

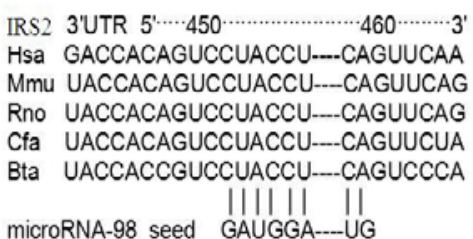

C

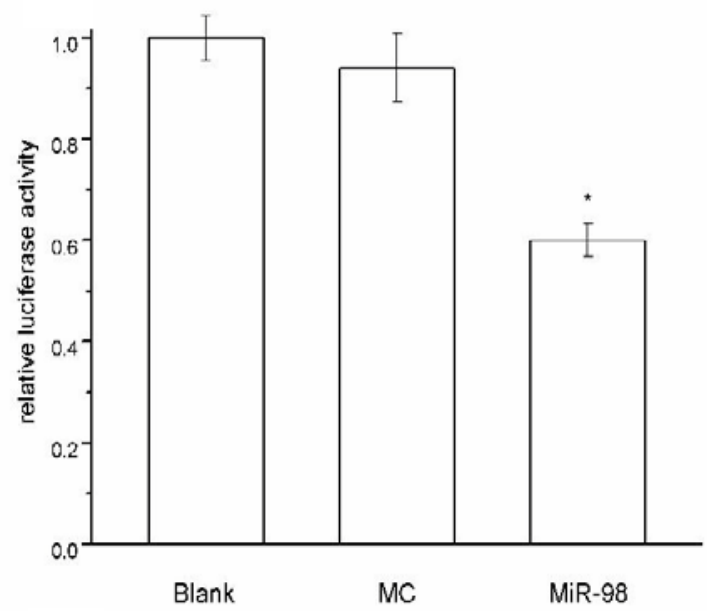

$\mathrm{B}$

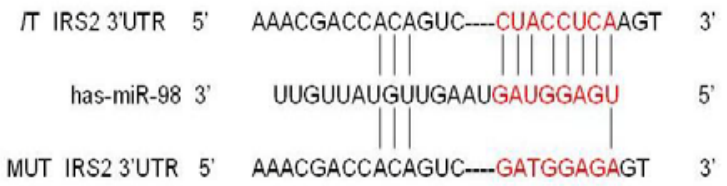

Figure 3. Targeting site of microRNA-98 in the 3'UTRof IRS2 and measurement ofits binding by repoter gene assay. Specific microRNA-98 binding to the 3'UTR ofIRS2.Two pGLO vector constructs, cotaining IRS-23'UTR-pmirGLO was transfected nto HEK-293Tcellseithor alone or in combination with miR-NTC or microRNA-98 minic. (A) Seed sequence of microRNA-98 is predicted to target IRS2 UTR and mut is stabilished at the same time. (B) Conservation of the microRNA-98 binding region in the IRS23'UTR between mammals, with the microRNA-98 seed match lighlighted in grey. (C) The repressive effect of microRNA-98 on the activity of IRS23'UTR measured by luciferase assay. (D) Mutation of the predicted microRNA-98 binding site in RS23'UTR abolished the repressive effect of microRNA-98 on the actvity of IRS23'UTR as measrued by luciferase assay. Values are expressed as mean \pm SEM from five independent experiments. ${ }^{*} \mathbf{p}<0.05$.

Meanwhile, insulin-induced glycogen synthase activity and subsequent glycogen synthesis in HepG2 cells were assayed by FACS analysis to observe the effect of miR-98 on glucose uptake. As shown in Figure 5, following insulin stimulation, IR hepatocytes exhibited a reduction in insulin-induced glucose uptake (approximately $36 \%$ 
reduction), which was later restored following treatment of the cells with miR-98 inhibitor (27\% recovery). These results clearly demonstrate that miR-98 can regulate IRS-2 protein levels and hepatic insulin sensitivity.

\subsection{Silencing the expression of miR-98 in-vivo restores hepatocyte IRS-2 levels and improves serum glucose levels}

Given that miR-98 can affect IRS-2 protein levels and insulin-induced pAkt activation as observed in the HepG2 cells, we sought to investigate whether miR-98 deficiency can protect against insulin resistance in vivo. After injection of miR-98 antagomir for silencing miR-98, we then measured random blood glucose and oral glucose tolerance levels. As shown in Figure 6A, the concentration of random blood glucose gradually decreased after injection compared with the $\mathrm{db} / \mathrm{db}$ mice that were injected with miR-98 scramble. Figure 6B shows the serum glucose levels after oral glucose tolerance test (OGTT). Db/db mice injected with miR-98 antagomir showed a peak increase in serum glucose concentration at 30min after glucose intake, but quickly reduced to normal levels within $120 \mathrm{~min}$. On the other hand, serum glucose concentration of $\mathrm{db} / \mathrm{db}$ mice injected with miR-98 scramble increased rapidly and remained at high levels even 120min after glucose intake. Finally, the animals were euthanized on the next day and liver tissue dissected for analysis. The levels of IRS-2, p-AKT and AKT were determined by Western Blot. As the results indicate (Figure 6C, 6D \& 6E), inhibition of miR-98 by antagomir significantly increased IRS-2 protein levels and insulin-induced phosphorylation of Akt in the liver of db/db mice.

A

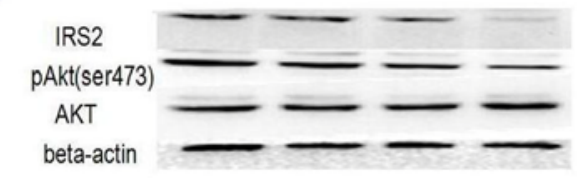

B

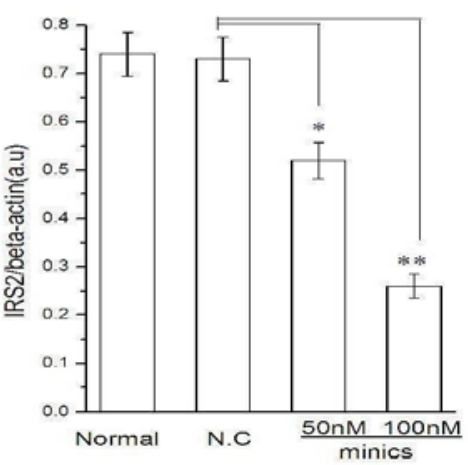

$\mathrm{C}$

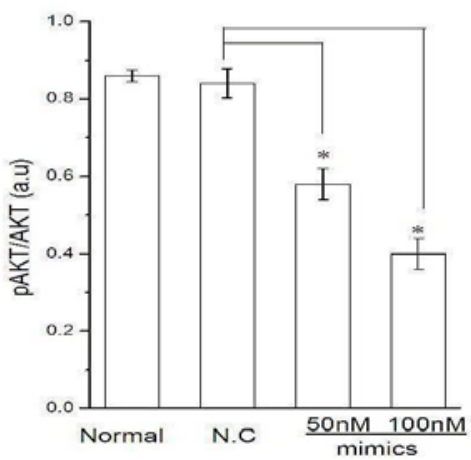

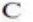

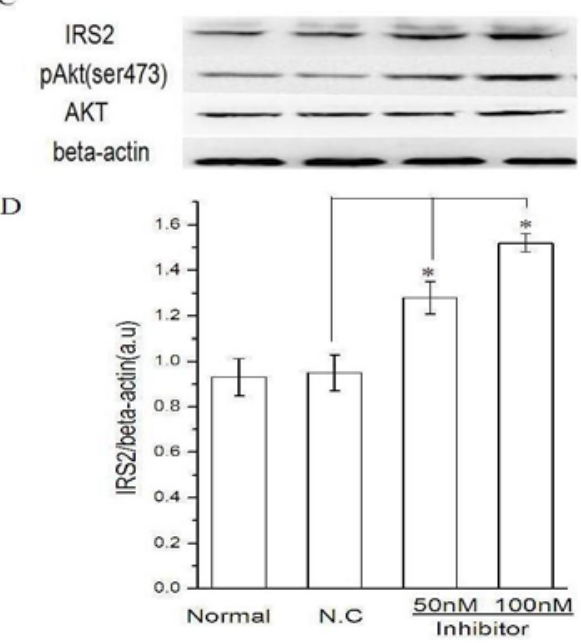

E

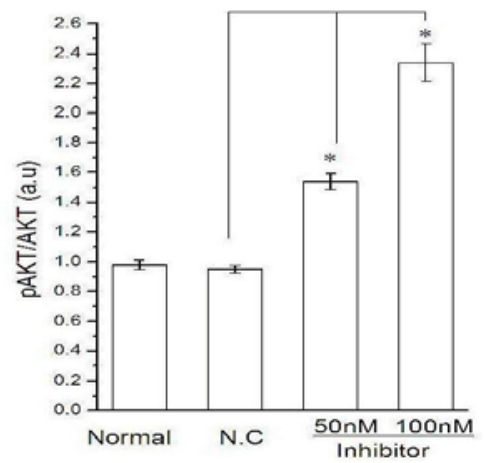

Figure 4. For insulin resistance, hepG2 cells were treated with insulin $(1 \times 10-6 \mathrm{molL})$ for $48 \mathrm{~h}$. And then, to indentified miR-98 can mediate the expression of IRS2, hepG2 cells were transfected with either the scramble (negative control) or miR-98 mimic(A B C) (5OnM, 100nM) or miR-98 mihibitor (DEF) (5OnM,100nM) and without any thing as normal. After transfection, cells were lysed and lysates (50ug) were representative of four such independent blots and the densitometric data is given below. The immunoblot intensities for IRS2. beta-actin subjected to Westem Blot analysis using IRS2, pAkt and Akt antibodies. Beta-actin was taken as the loading control. Each blot is a was quantified by densitometry and expressed in relative ratio where the intensity of negative control was set to one. The immunoblo intensities for pAkt Akt was quantified by densitometry and expressed in relative ratio. The intensity of normal control was set to one. Values are expressed as means \pm SEM from five independent experiments, ${ }^{*} \mathbf{P}<0.05 ;{ }^{* *} \mathbf{P}<0.001$. 


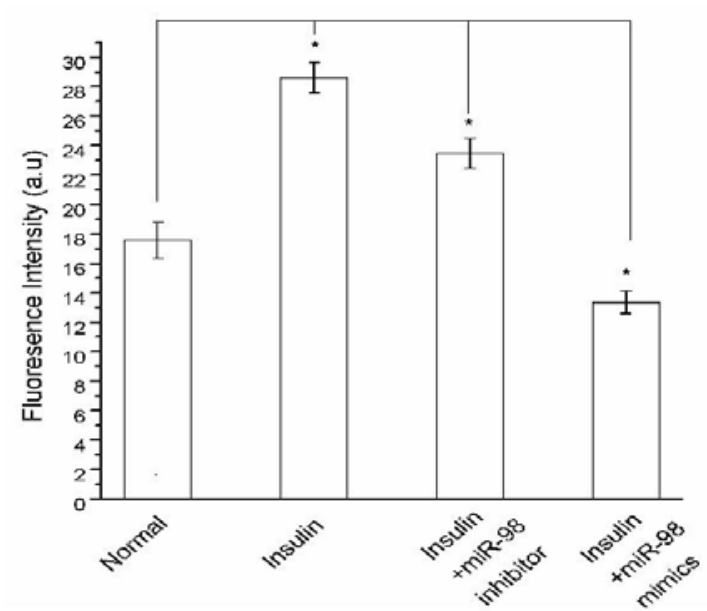

Figure 5. To demonstrate the effect of miR-98 mimics and its inhibitor on glucose uptake into hepG2 cells which treated with insulin, cells were transfected with the scramble or miR-98 mimics or its inhibitor. After 48h, cells were treated with 2-NBDG (30OuMD) for $1 \mathrm{~h}$ at 37C and washed by PBS for three times. And then the 2-NBDG uptake into the cell was evaluated by FACS analysis. Values are expressed as means \pm SEM from five independent experiments ${ }^{*} \mathbf{P}<0.05$.

A
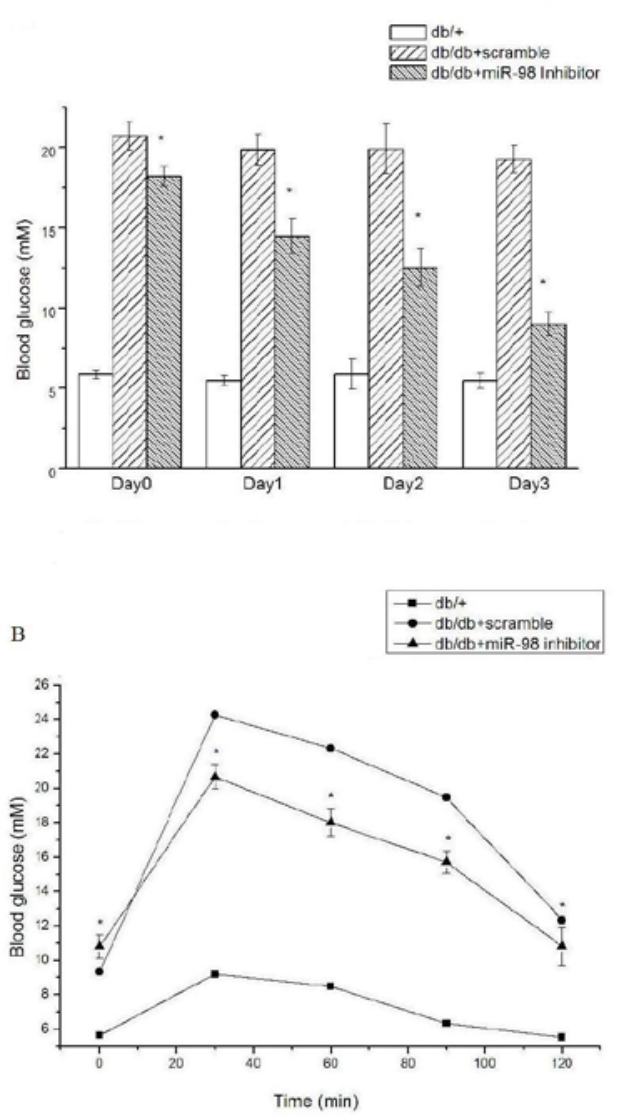
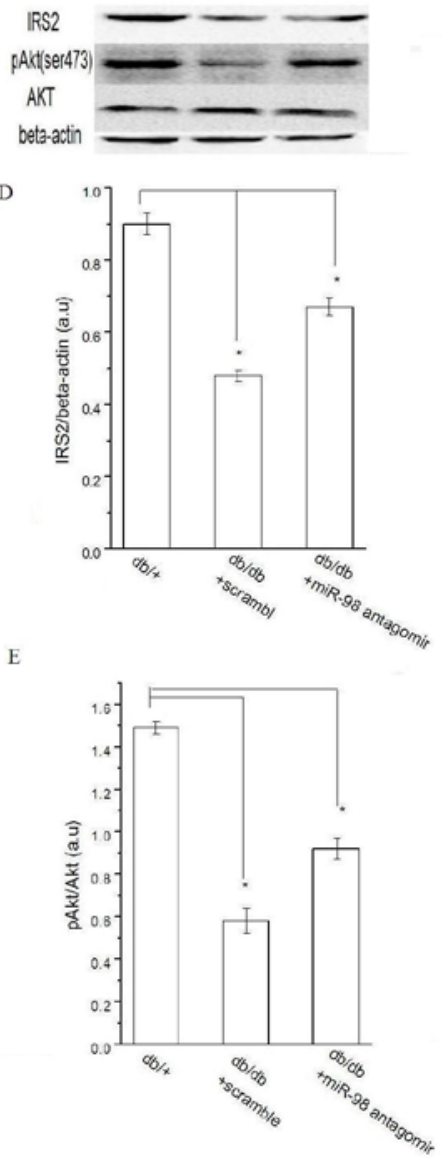

Figure 6. To identify silencing of miR-98 in wivo can alleviate hyperglyemia and upregulate the expression of IRS2 protein in Diabetic dbldb mice and $\mathrm{db} /+$ mice( $n=5$ in each group). They were injected with either the scramble or the miR.-98 antagomir. (A) Random blood glucose levels were measured from Oday to the third day on each day ofinjection. (B) Oral glucose tolerance test (OGTT) was done after three days of injection. (C) Livertssues were dissected from each mice and leves of IRS2, pAkt and Akt were detected by western blot. Beta-actin was used as the loading control. A representative blot is shown and the densitometric analysis is below. (D, E) Data presented as mean \pm SEM with $n=5$ for each group. Statistically significant differences are test at $\mathbf{p}<0.05$ significance. ${ }^{*} \mathbf{p}<0.05$. 


\section{Conclusion}

We infirmed that microRNA-98 was related to bariaitric surgey and it was up-regulated in $\mathrm{db} / \mathrm{db}$ mice, and played a key role in insulin resistance by inhibiting IRS-2 protein expression. These findings provide novel insights into the molecular basis of insulin resistance, and implicate bariatric surgery may change change the expression of microRNA to improve insulin resistance.

\section{Discussion}

In recent years, microRNAs have emerged as important regulators in insulin signaling $[19,20]$. Several microRNAs have been shown to target the 3'-untranslated regions of mRNAs encoding components of the insulin signaling pathway. MicroRNA-143 over-expression was shown to be induced by obesity, and in turn, microRNA-143 acted as a negative regulator to insulin signaling by inhibiting insulin-induced AKT activation, and impairing glucose metabolism in liver cells. It contributes to the development of obesity-induced insulin resistance [21].

Insulin signal transduction is achieved by binding to a specific tyrosine kinase receptor expressed in target cells, resulting in increased phosphorylation and activation of substrate proteins, and complex second messenger cascades that regulate glucose and lipid metabolism [22]. Insulin receptor substrate (IRS) proteins are a family of molecules that are directly phosphorylated by the insulin receptor [23]. IRS proteins play a crucial role in insulin action, as has been demonstrated in this study. Reduction of IRS expression and/or function may be a fundamental step in the development of insulin resistance in T2DM [24]. The expression of IRS proteins can be mediated by microRNAs, as has been demonstrated by previous studies. McroiRNA-26 has been observed to repress translation of IRS-1 without altering the mRNA level of IRS-1, which subsequently leads to insulin resistance in hepatocytes [25]. Dwi, Setyowati, Karolina et al demonstrated that microRNA-144 could also impair insulin signaling by inhibiting the expression of IRS-1 in T2DM [26]. We therefore propose the hypothesis that certain microRNAs may participate in the development of insulin resistance by altering the expression of key proteins involved in the insulin signaling pathway.

The first part of our investigation revealed that down-regulate the expression of miR-98 in the liver after 30 days of bariatric surgery.And then we found that miR-98 was significantly up-regulated in the hepatocytes of $\mathrm{db} / \mathrm{db}$ mice compared with the $\mathrm{db} /+$ control mice. MiR-98 had previously been reported in earlier studies and was notably up-regulated during adipogenesis [27]. In table 1, we can clearly understand that bariatric surgery is effective to improve blood glucose tolerance and insulin resistance in $\mathrm{db} / \mathrm{db}$ mice. What is the relationship between miR-98 and remission of leve of blood glucose after surgery?

We also demonstrated that IRS-2 levels in $\mathrm{db} / \mathrm{db}$ mice were significantly decreased compared with $\mathrm{db} /+$ mice. At the same time, using TargetScan, PicTar and miRBase, we discovered that miR-98 bound to IRS-2 3'UTR at eight different binding sites. Based on these findings, we have proposed a mechanism by which miR-98 can potentially control the expression of IRS-2, and how its regulatory effect governs the insulin signaling pathway in the pathogenesis of T2DM. Subsequent functional analysis with 293T cells proves that miR-98 decreases IRS-2 levels by binding to its 3'UTR. The luciferase activity of the IRS-2 3'UTR was significantly decreased in the presence of miR-98. This decrease was markedly blunted in the presence of miR-98 inhibitor or a mutation in the miR-98 binding site. To the best of our knowledge, this is the first time IRS-2 is being highlighted as a key target for miR-98. As we know, activation of IRS-2 by binding of insulin to its receptor initiates a signaling cascade transmitted through the PI3K/Akt pathway, which subsequently facilitates glucose uptake into the cell [28]. So we measured the levels of IRS-2, Akt, pAkt and glucose uptake in HepG2 cells treated with insulin after transfection. As our results indicate, in the presence of miR-98, insulin-induced Akt phosphorylation was significantly attenuated. Similarly, when we up-regulate the expression of miR-98, the level of Akt phosphorylation was also decreased. The uptake of glucose varied with up-regulation or down-regulation of miR-98 in HepG2 cells. Together, these results confirm that miR-98 can regulate insulin signaling and glucose uptake in cells.

However, whether the effect of miR-98 on insulin sensitivity is also IRS-2-dependent in vivo remained unclear. After injection of $\mathrm{db} / \mathrm{db}$ mice with miR-98 antagomir, we found that the level of IRS-2 and pAkt were markedly increased compared with $\mathrm{db} / \mathrm{db}$ mice injected with miR-98 scramble. At the same time, the hyperglycemic status of $\mathrm{db} / \mathrm{db}$ mice was also improved. These observations indicate that miR-98 may play a dominant role in the regulation of insulin signaling in vivo. In a recent study, Lei Cao et al demonstrated that microRNA can cure obesity-induced insulin resistance in $\mathrm{db} / \mathrm{db}$ mice. According to their findings, microRNA can mimic the body's endogenous physiological feedback mechanism, thereby resetting the hypothalamic set point to reverse obesity and 
other metabolic syndromes by stimulating brain-derived neurotrophic factor (BDNF), a key element in the regulation of energy balance [29].

In conclusion, our results indicate that miR-98 regulates hepatic insulin sensitivity, and that IRS-2 is involved in this process. Inhibition of miR-98 can improve hepatic insulin sensitivity and glucose homeostasis, potentially providing a new mechanism for bariatric surgery to treat insulin resistance and T2DM.

\section{Disclosure}

The authors of this article declare no conflict of interest. All experiments were performed in compliance with the local animal ethics committee.

\section{References}

[1] Annette Boles, Ramesh, Kandimalla, P Hemachandra Reddy. (2017). Dynamics of diabetes and obesity: Epidemiological perspective.Biochim Biophys Acta Mol Basis Dis. 2017 May; 1863(5): 1026-1036.

[2] D. J. Kim, M. S. Lee, K. W. Kim, M. K. Lee. (2001). Insulin secretory dysfunction and insulin resistance in the pathogenesis of korean type 2 diabetes mellitus. Metabolism. 2001 May; 50(5): 590-3.

[3] Shulman, G. I. (2000). Cellular mechanisms of insulin resistance. J Clin Invest. 2000 Jul; 106(2): 171-6.

[4] Saltiel, A. R., and Kahn, C. R. (2001). Insulin signalling and the regulation of glucose and lipid metabolism. Nature. 2001 Dec 13; 414(6865): 799-806.

[5] Tetsuya Kubota, Naoto Kubota, Takashi Kadowaki. (2017). Imbalanced Insulin Actions in Obesity and Type 2 Diabetes: Key Mouse Models of Insulin Signaling Pathway. Cell Metab. 2017 Apr 4; 25(4): 797-810.

[6] Saltiel, A. R., Pessin, J. E. (2002). Insulin signaling pathways in time and space. Trends Cell Biol. 2002 Feb; 12(2): 65-71.

[7] Hiroyuki Yano, Mashito Sakai, Toshiya Matsukawa, Takashi Yagi, Takao Naganuma, et al. (2018). PHD3 regulates glucose metabolism by suppressing stress-induced signalling and optimising gluconeogenesis and insulin signalling in hepatocytes.Sci Rep. 2018 Sep 24; 8(1): 14290.

[8] Choudhury, A. I., Heffron, H., Smith, M. A., Al-Qassab, H., Xu, A. W., Selman, C., et al. (2005). The role of insulin receptor substrate 2 in hypothalamic and $\beta$ cell function. J. Clin. Invest. 2005 Apr; 115(4): 940-50. Epub 2005 Mar 24.

[9] Farh, K. K., Grimson, A., Jan, C., Lewis, B. P., Johnston, W. K., Lim, L. P. (2005). The widespread impact of mammalian MicroRNAs on mRNA repression and evolution. Science. 2005 Dec 16; 310(5755): 1817-21. Epub 2005 Nov 24.

[10] Flynt, A. S., Lai, E. C. (2008). Biological principles of microRNA-mediated regulation: shared themes amid diversity. Nat Rev Genet. 2008 Nov; 9(11): 831-42.

[11] Leeper, N. J., Cooke, J. P. (2011). MicroRNA and mechanisms of impaired angiogenesis in diabetes mellitus. Circulation, 2011 Jan 25; 123(3): 236-8. Epub 2011 Jan 10.

[12] Yury O Nunez Lopez, Gabriella Garufi, Attila A Seyhan. (2016). Altered levels of circulating cytokines and microRNAs in lean and obese individuals with prediabetes and type 2 diabetes. Mol Biosyst. 2016 Dec 20; 13(1): 106-121.

[13] Spriggs, K. A, Bushell, M., Willis, A. E. (2010). Translational regulation of gene expression during conditions of cell stress. Mol Cell. 2010 Oct 22; 40(2): 228-37.

[14] Raitoharju, E., Seppälä, I., Oksala, N., Lyytikäinen, L. P., Raitakari, O., Viikari, J., et al. (2014). Blood microRNA profile associates with the levels of serum lipids and metabolites associated with glucose metabolism and insulin resistance and pinpoints pathways underlying metabolic syndrome: the cardiovascular risk in Young Finns Study. Mol Cell Endocrinol. 2014 Jun 25; 391(1-2): 41-9.

[15] Obert J, A. Frost Eric, N. Olson, et al. (2011). Control of glucose homeostasis and insulin sensitivity by the Let-7 family of microRNAs. Proc Natl Acad Sci USA. 2011, 27; 108(52): 21075-80.

[16] Xie, S., Xie, N., Li, Y., et al. (2012). Upregulation of TRB2 induced by miR-98 in the early lesions of large artery of type-2 diabetic rat. Mol Cell Biochem. 2012; 361(1-2): 305-14.

[17] David E. Cummings, Francesco Rubino. (2018). Metabolic surgery for the treatment of type 2 diabetes in obese individuals.Diabetologia. 2018 Feb; 61(2): 257-264.

[18] Chen, H., Charlat, O., Tartaglia, L. A., Woolf, E. A., Weng, X., Ellis, S. J., et al. (1996). Evidence that the diabetes gene encodes the leptin receptor: identication of a mutation in the leptin receptor gene in db/db mice. Cell, 1996 Feb 9; 84(3): 491-5.

[19] Kornfeld, J. W., Baitzel, C., Könner, A. C., Nicholls, H. T., Vogt, M. C., Herrmanns, K., et al. (2013). Obesity-induced overexpression of miR-802 impairs glucose metabolism through silencing of Hnf1b. Nature. 2013 Feb 7; 494(7435): 111-5.

[20] Yun-Feng Zhen,Yun-Jia Zhang, Hang Zhao, et al. (2018). MicroRNA-802 regulates hepatic insulin sensitivity and glucose metabolism. Int J Clin Exp Pathol. 2018 May 1; 11(5): 2440-2449.

[21] Jordan, S. D., Krüger, M., Willmes, D. M., Redemann, N., Wunderlich, F. T., Brönneke, H. S., et al. (2011). Obesiy-induced overexpression of miRNA-143 inhibits insulin-stimulated AKT activation and impairs glucose metablolism. Nat Cell Biol. 
2011 Apr; 13(4): 434-46.

[22] Saltiel, A. R., and Kahn, C. R. (2001). Insulin signalling and the regulation of glucose and lipid metabolism. Nature. 2001 Dec 13; 414(6865): 799-806.

[23] Tamemoto, H., Kadowaki, T., Tobe, K., Yagi, T., Sakura, H., Hayakawa, T., et al. (1994). Insulin resistance and growth retardation in mice lacking insulin receptor substrate-1. Nature. 1994 Nov 10; 372(6502): 182-6.

[24] Withers, D. J., Gutierrez, J. S., Towery, H., Burks, D. J., Ren, J. M., Previs, S., et al. (1998). Disruption of IRS-2 causes type 2 diabetes in mice. Nature. 1998 Feb 26; 391(6670): 900-4.

[25] Ryu, H. S., Park, S. Y., Ma, D., Zhang, J., Lee, W. (2011). The Induction of MicroRNA Targeting IRS-1 Is Involved in the Development of Insulin Resistance under Conditions of Mitochondrial Dysfunction in Hepatocytes. PLoS One. 2011 Mar 25; 6(3): e17343.

[26] Karolina, D. S., Armugam, A., Tavintharan, S., Wong, M. T., Lim, S. C., Sum, C. F., et al. (2011). MicroRNA 144 Impairs Insulin Signaling by Inhibiting the Expression of Insulin Receptor Substrate 1 in Type 2 Diabetes Mellitus. PLos One. 2011; 6(8): e22839.

[27] Kajimoto, K., Naraba, H., Iwai, N. (2006). MicroRNA and 3T3-L1 preadipocyte differentiation. RNA 2006. $12,1626 \mathrm{e} 1632$.

[28] L. F. del Aguila, K. P. Claffey, J. P. Kirwan. (1999). TNF-alpha impairs insulin signaling and insulin stimulation of glucose uptake in C2C12 muscle cells. Am. J. Physiol. Am J Physiol. 1999 May; 276(5 Pt 1): E849-55.

[29] Cao, L., Lin, E. J., Cahill, M. C., Wang, C., Liu, X., During, M. J. (2009). Molecular therapy of obesity and diabetes by a physiological autoregulatory approach. Nat Med. 2009 Apr; 15(4): 447-54. 